\title{
Minimizing bushfire risk through optimal powerline assets replacement and improvement
}

\author{
R. Roozbahani ${ }^{\text {a }}, \underline{\text { C. Huston }}^{\text {a }}$, S. Dunstall ${ }^{\text {a }}$, Babak Abbasi ${ }^{\text {b }}$, A. Ernst ${ }^{\text {a }}$ and S. Schreider $^{\text {c }}$ \\ ${ }^{a}$ Digital Productivity Flagship CSIRO, Clayton, Victoria, 3169 \\ ${ }^{\mathrm{b}}$ RMIT School of Business IT and Logistics, \\ ${ }^{\mathrm{c}}$ RMIT School of Math and Geospatial Sciences \\ Email: carolyn.huston@csiro.au
}

\begin{abstract}
Fires initiated by powerline faults disproportionately are associated with a majority of bushfire fatalities in South-Eastern Australia. Over 150 deaths have occurred since 1977 in South-Eastern Australia. A response from governments and utilities has been to embark on electricity asset improvement and replacement programs where the definition of improvement is tied to an aim of reducing powerline sparked ignitions under the most dangerous meteorological conditions for fire. This paper introduces an optimization model which provides a strategy for adding technologies to powerline distribution assets such that there are improvements in terms fire risk being lowered. The goal of the model is to minimize financial outlay while the risk of electricity sparked bushfire incidence is a solid constraint in the model, set to mimic potential bushfire risk reduction target scenarios. Currently, strategies for targeting investment in improvement of the electrical distribution system have not used such a mathematically based optimization approach. Instead they are based on expert interpretation of risk maps which visualize risk of fault of asset technologies with the consequence of fire starts at the same locations. Application of an optimization model by government and utilities when investing in powerline improvements could lead to reduced bushfire impacts within given funding frameworks relative to current practice. Estimated fault and fire ignition behavior of current and proposed electrical asset technology are a basis for our model. Fire mitigating treatments can range from the installation of new electrical fault detection systems at zone sub-stations; burying individual sections of powerline; installation of automatic circuit reclosers (ACRs); adjusting the settings on existing ACRs; insulating bare lines; etc. The work here represents a natural extension of bushfire risk-modelling work being undertaken by the authors in collaboration with the Victorian Government's Powerline Bushfire Safety Program (PBSP).
\end{abstract}

Keywords: Bushfire risk, mixed-integer programming, powerline safety, combinatorial optimization 


\section{INTRODUCTION}

Bushfire is an ever present risk to life, property and the environment, primarily in rural areas. Although the number of fires triggered by powerlines in comparison to other causes is low, around 14 percent in any given year (Energy Safe Victoria, 2011), they are a key cause of major Victorian bushfires that occurred on February 12, 1977; on February 16, 1983 (Ash Wednesday); and on February 7, 2009 (Black Saturday) (Energy Safe Victoria, 2011). The Black Saturday fires resulted in a Bushfires Royal Commission in Victoria found that on Black Saturday, electrical faults caused five of the 11 major fires (Teague et al., 2010). Black Saturday resulted in 173 fatalities and damage to property, infrastructure and the natural environment with an estimated total cost of over AU \$4 billion (Teague et al., 2010). The determination that powerlines were a significant cause contributing to such large bushfires led the Royal Commission to make a number of recommendations on investigating and mitigating electrical distribution started bushfire risk. These risk reduction measures include improved maintenance of existing electrical infrastructure and installing new technology to areas with high bushfires risk (Teague et al., 2010). Currently electrical asset replacement and improvements are selected on the basis of available budget and a combination of expert judgement and scientific input. Risk is defined as the rate of ignition at a particular location multiplied by potential consequence at that location under high fire condition exposure. We can compare the relative bushfire risk reductions of different network asset treatment combinations and locations to compare different improvement approaches.

We propose an optimization model for investigating improvements/replacements of current electricity assets in powerline networks. Optimization techniques have widely been used for managing disasters such as hurricanes, earthquakes, and floods (Altay and Green, 2006). They have also been applied to other wildfire management problems. Hof et al. (2000) introduced a timing-oriented optimization model based on linear programming for the spatial allocation of suppression effort for an existing fire. Minciardi et al. (2009) presented two nonlinear optimization models, one for deployment of wildfire suppression resources in the pre-operational phase and the other for dispatch of resources to fires in the operational phase. Higgins et al. (2011) formulated an integer program (IP) for planning fuel reduction burning on public lands in Victoria, Australia. Minas et al. (2013) developed an IP model for integrating suppression preparedness decisions and fuel management in forest fire management.

Here, an optimization model is introduced for investigating the optimal improvements/replacements of current electricity assets in powerline networks that lead to a particular reduction in the bushfires risks caused by electricity assets in the networks. We formulate a mixed-integer program (MIP). The objective function of the model is to minimize the cost of improvements/replacements. A key constraint is a bushfire risk threshold applied across the network. The model was evaluated on an example network similar to those found in Victoria. To the best of our knowledge, this is the first study aimed to reduce bushfires risk posed by an electrical network linked to a risk threshold with lowest possible cost.

\section{MinimuM COST (MC) MODEL FORMULATION}

The MC model is a mixed-integer linear programming model and it is based on a tree network structure. Poles are fed by sections and sections belong to zone substations. Zone substations make the root of the trees. Figure 1 illustrates the schematic of a tree network.

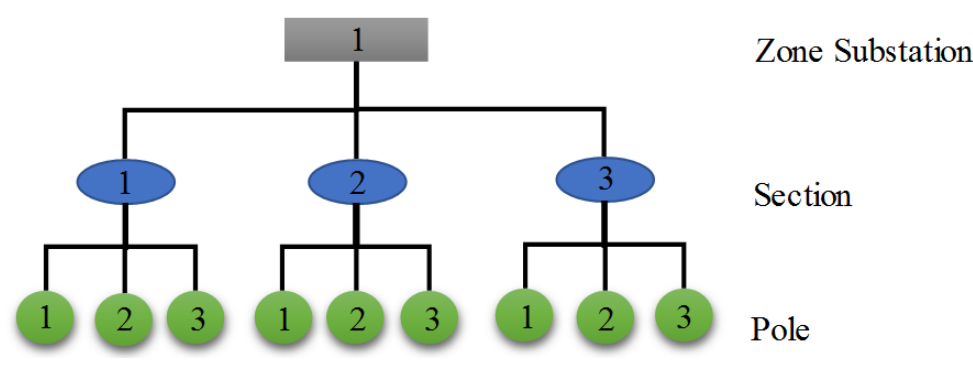

Figure 1. The schematic of a tree network in a powerline grid 
R.Roozbahani et al., Minimizing bushfire risk through electrical asset replacement

\title{
2.1 Notation
}

Sets and indices.

$k$ : is zone substation $(1, \ldots, K) ; \quad \Omega$ : is the set of zone subsections in a network;

$j$ : is section $(1, \ldots, J) ; \quad \tau^{\prime \prime}:$ is treatment for a pole $\left(1, \ldots, \Psi^{\prime \prime}\right)$;

$i$ : is pole $(1, \ldots, I) ; \quad \tau^{\prime}:$ is treatment for a section $\left(1, \ldots, \Psi^{\prime}\right) ;$

$a$ : is an asset combination $(a \in A) ; \quad \tau$ : is the treatment for a zone $\operatorname{substation}(1, \ldots, \Psi)$;

$A$ : is the set of asset combinations; $\quad \Phi_{k}$ : is the set of sections in zone substation $k$

$\Pi_{j}$ : is the set of poles in section $j ; \quad O_{a}$ : is the set of treatment for poles, $\tau^{\prime \prime}$, sections, $\tau^{\prime}$, and

zone subsections, $\tau$, associated with asset combination $a$

\section{Input and modelling parameters.}

$C_{i j k \tau^{\prime \prime}}^{p}:$ is the cost of applying treatment $\tau^{\prime \prime}$ to pole $i$, located in section $j$ and zone substation $k$;

$$
\begin{array}{r}
C_{j k \tau^{\prime}}^{S}: \text { is the cost of applying treatment } \tau^{\prime} \text { to } \\
\text { section } j \text {, located at zone substation } k \text {; }
\end{array}
$$
$C_{k \tau}^{Z}$ : is the cost of applying treatment $\tau$ to zone subsection $k$; $f_{i j k}$ : is fires loss consequence caused by a fault at pole $i$, located in section $j$ and zone substation $k$ which results in a bushfire;

$R_{i j k}$ : is the existing likelihood of ignition at pole $i$, located in section $j$ and zone substation $k$; $\eta_{a}$ : is the reduction in ignition likelihood at a pole if combination $s$ is applied to a network.

\section{Decision variables.}

\author{
$\delta_{i j k \tau^{\prime \prime}}^{p}:$ is a binary variable set to 1 \\ $\delta_{k \tau}^{Z}$ : is a binary variable set to 1 if treatment $\tau$ \\ if treatment $\tau^{\prime \prime}$ is applied to pole $i$ located \\ will be applied to zone substation $k$; \\ in section $j$ and zone substation $k$; \\ $\delta_{j k \tau^{\prime}}^{S}:$ is a binary variable set to 1 if treatment $\tau^{\prime}$ \\ $\varphi_{a}^{i j k}$ : is a binary variable set to 1 if asset \\ is applied to section $j$ located in zone substation $k$; \\ combination $a$ is applied to the network
}

\section{Exogenous variables.}

$T C$ : is the total cost of improvements

to powerline assets; $C^{S}$ : the total cost of improvements of sections on the network;

$C^{Z}$ the total cost of improvements of zone substations on the network;
$C^{p}$ the total cost of improvements to poles on the network; $r_{i j k}$ : the new likelihood of fault at pole $i$ located in section $j$ and zone substation $k$ for creating a bushfire, considering improvements.

\subsection{MC model objective function}

When minimizing cost, the MC model formulation aims to achieve a particular threshold for fire risk with the lowest cost. Thus, the objective function of $\mathrm{MC}$ is to minimize the cost of asset improvements/replacements on the entire network. This can be expressed as 


$$
\begin{aligned}
T C=C^{p}+C^{S}+C^{Z} & C^{p}=\sum_{k=1}^{K} \sum_{j=1}^{J} \sum_{i=1}^{I} \sum_{\tau^{\prime \prime}=1}^{\Psi^{\prime \prime}} C_{i j k \tau^{\prime \prime}}^{p} \times \delta_{i j k \tau^{\prime \prime}}^{p} \\
C^{s}=\sum_{k=1}^{K} \sum_{j=1}^{J} \sum_{\tau^{\prime}=1}^{\Psi^{\prime}} C_{j k \tau^{\prime}}^{s} \times \delta_{j k \tau^{\prime}}^{s} & C^{Z}=\sum_{k=1}^{K} \sum_{\tau=1}^{\Psi} C_{k \tau}^{z} \times \delta_{k \tau}^{z}
\end{aligned}
$$

\subsection{MC model constraints}

The objective function of the MC model is subjected to several constraints. These constraints are summarized below.

$$
\begin{aligned}
& \sum_{k=1}^{K} \sum_{j=1}^{J} \sum_{i=1}^{I} f_{i j k} \times r_{i j k} \leq F \\
& \sum_{\tau^{\prime \prime}=1}^{\Psi^{\prime \prime}} \delta_{i j k \tau^{\prime \prime}}^{p}=1 \forall i, j, k \\
& \sum_{\tau^{\prime}=1}^{\Psi^{\prime}} \delta_{j k \tau^{\prime}}^{s}=1 \forall j, k \\
& \sum_{k \tau}^{\Psi} \delta_{k \tau}^{z}=1 \forall k \\
& \sum_{a=1}^{A} \varphi_{a}^{i j k}=1 \forall i, j, k \\
& r_{i j k}=R_{i j k} \times\left[1-\sum_{a \in A}\left(\eta_{a} \times \varphi_{a}^{i j k}\right)\right] \quad \forall i \in \Pi_{j}, j \in \Phi_{k}, k \in \Omega \\
& \delta_{k \tau}^{z} \geq \varphi_{a}^{i j k} \quad \forall i \in \Pi_{j}, j \in \Phi_{k}, k \in \Omega, a \in A, \tau \in O_{a} \\
& \delta_{j k \tau^{\prime}}^{s} \geq \varphi_{a}^{i j k} \quad \forall i \in \Pi_{j}, j \in \Phi_{k}, k \in \Omega, a \in A, \tau^{\prime} \in O_{a} \\
& \delta_{i j k \tau^{\prime \prime}}^{p} \geq \varphi_{a}^{i j k} \quad \forall i \in \Pi_{j}, j \in \Phi_{k}, k \in \Omega, a \in A, \tau^{\prime \prime} \in O_{a}
\end{aligned}
$$

Equation 2 expresses the bushfire risk for the entire network after taking into consideration improvements to poles, sections and zone substations. In this case, risk is defined as the risk of a fault causing an ignition under given environmental conditions. Consequence is also considered in the model, where consequence measures the expected amount of damage based on an ignition at a location. The goal is to reduce the bushfire risk of the network to less than a threshold $(F)$. It is impractical to make the risk of bushfire in the network zero due to the huge financial investment that would be required. Consequently the value of $F$ needs to be pre-determined.

Equations 3, 4 and 5 express that the model can only select one treatment from the multiple potential choices for each pole, section, and zone substation. For this purposes, the binary variables $\delta_{i j k \tau^{\prime \prime}}^{p}, \delta_{j k \tau^{\prime}}^{s}$ and $\delta_{k \tau}^{z}$ are introduced to the model. For instance, if treatment $\tau^{\prime \prime}$ is applied to pole $i$ the value of $\delta_{i j k \tau^{\prime \prime}}^{p}=1$, and is otherwise 0 . Similarly for $\delta_{j k \tau^{\prime}}^{s}$ and $\delta_{k \tau}^{z}$. It needs to be noted that in this study, the amount of ignition likelihood reduction for a pole is available in the form of asset combinations (a set of treatments for a pole and its related section and zone substation). For instance, it is given that the ignition likelihood on a pole would decrease $60 \%$ if treatment $\tau^{\prime \prime}$ on the pole, $\tau^{\prime}$ on the section, and $\tau$ on its zone substation are applied. Equation 6 forces the model to select only one asset combination for node $i$ located in section $j$ and zone substation $k$. The binary variable $\varphi_{a}^{i j k}$ is set to 1 if asset combination $a$ will be applied to pole $i$, otherwise it is 0 . Constraint 7 is imposed to determine the new likelihood of faults at poles after improvements/replacements on poles and their associated sections and zone substations. The new likelihood is calculated based on the existing likelihood of ignitions at poles $\left(R_{i j k}\right)$ and the amount of likelihood reduction $\left(\eta_{a}\right)$ by applying asset combination $a$. According to the tree structure of the network, the model should select the asset combinations for all poles in section $j$ in a way that the treatment of section $j$ will be the same for all combinations. Similar constraints need to be considered for zone substations, and are actioned by constraints 8, 9, and 10. For example, Equation 8 illustrates that the value of binary variable $\delta_{k \tau}^{Z}$ indicates the treatment on zone substation 


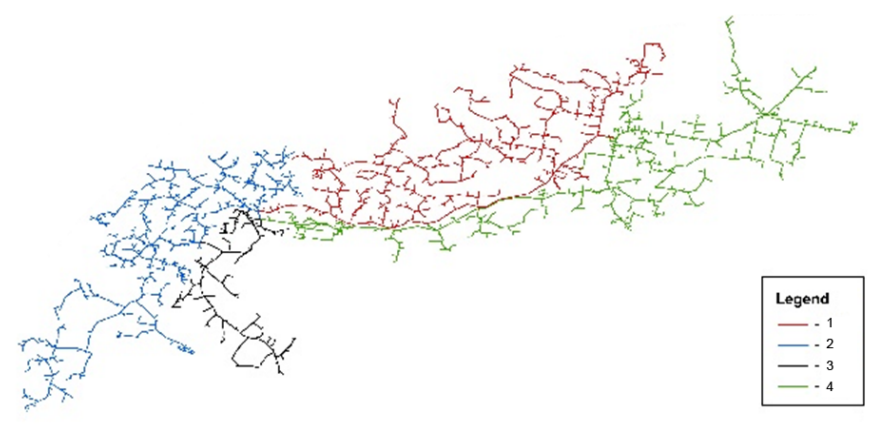

Figure 2. Mapping of the case study network with colours indicating zone substation areas of influence

$k$ has to be greater than or equal to binary variable $\varphi_{a}^{i j k}$ which screens the asset combinations. In practice, this means that an asset applied to a zone substation needs to be reflected in the potential treatments considered for downstream sections and poles.

\section{IMPLEMENTATION}

This section briefly introduces the case study and some information about the input parameters for the model as associated with the case study.

\subsection{Case Study}

The proposed optimization model was applied to a demonstration network consistent with the type of assets and deployments observed on the Victorian powerline network. The case study network consists of 4 zone substations that we label 1 through 4 . The demonstration network is illustrated in Figure 2. To initiate the optimization it is assumed that all lines in the network are bare wire and no new technologies have been installed on zone substations, sections, and poles.

\subsection{MC inputs}

The existing likelihood of ignitions were obtained from Dunstall et al. (2015). In this, likelihood quantification relied on data gathered from distribution network records of faults and ignitions. Estimates were generated for existing asset types according to their performance related to a variety of environmental conditions including terrain, vegetation/fuel type, wind, temperature, etc. Fault rates for different possible combinations of environmental and asset variables were calculated. The proportion of faults leading to ignitions was also estimated, assuming it was dependent on the occurrence of a fault and the fire danger index (FDI) at the time of the fault. There were too few ignitions for the ignition model to be directly based on the larger number of environmental conditions considered for faults. To create conservative asset improvement suggestions, this study assumed worst case fire conditions, and other conditions were obtained assuming the demonstration network was located in south-eastern Victoria.

A number of different treatments for zone substations, sections, and poles are considered in this study. Only treatments that were considred practically feasible given the physical network structure and hierarchy are shown, resulting in 12 options. These include rapid earth fault current limiter (REFCL) and high impedence (HiZ) zone substation treatments, and a baseline of no treatment. Section treatments considered are the addition of automatic circuit reclosers (ACRs). Three different pole/line treatments were considered: insulated overhead wire and bare pole equipment (IOBE); insulated overhead wire and insulated equipment (IOIE); and underground wire and insulated equipment (UIE). When none of the above treatments was applied a no treatment (NT) designation was given. Table 1 introduces asset combinations and their associated fault likelihood reductions if they are applied to the network. The amount of risk reduction for various asset combinations was estimated in Dunstall et al. (2015). Table 1 also presents the approximate costs of applying various treatments to zone substations, sections, and poles. The level of bushfire risk reduction that the model aimed to achieve was a constraint in the model. To understand how different risk reduction targets would affect strategy, we ran ensembles of the optimization model using risk reduction targets $(F)$ selected sequentially between $10 \%$ and $90 \%$. 
Table 1. Proposed asset combinations, treatment costs, and assumed fault rate reductions. Costs for Z.T. are given in $\$ 1000$; costs for S.T. are given in $\$ 1000$ plus $\$ 1000$ per metre line; and P.T costs are given as the cost for insulating equipment plus 1 meter wire insulating or undergrounding

\begin{tabular}{llllllll}
\hline $\begin{array}{l}\text { Asset } \\
\text { combina- } \\
\text { tion }\end{array}$ & $\begin{array}{l}\text { Zone } \\
\text { substation } \\
\text { treatment } \\
(\text { Z.T. })\end{array}$ & $\begin{array}{l}\text { Cost Z.T. } \\
(\$ 1000)\end{array}$ & $\begin{array}{l}\text { Section } \\
\text { Treatment } \\
(\text { S.T. })\end{array}$ & $\begin{array}{l}\text { Cost S.T. } \\
(\$ 1000)\end{array}$ & $\begin{array}{l}\text { Pole } \\
\text { Treatment } \\
(\text { P.T. })\end{array}$ & $\begin{array}{l}\text { Cost } \\
(\$)\end{array}$ & $\begin{array}{l}\text { P.T. } \\
\text { Fault } \\
\text { likelihood } \\
\text { reduction } \\
(\%)\end{array}$ \\
\hline 1 & NT & 0 & ACR & 60 & NT & 0 & 20.5 \\
\hline 2 & NT & 0 & HiZ & 5 & NT & 0 & 8.1 \\
\hline 3 & NT & 0 & ACR\&HiZ & $60+5$ & NT & 0 & 28.7 \\
\hline 4 & REFCL & 4000 & NT & 0 & NT & 0 & 57 \\
\hline 5 & REFCL & 4000 & ACR & 60 & NT & 0 & 62.9 \\
\hline 6 & REFCL & 4000 & ACR\&HiZ & $60+5$ & NT & 0 & 68.6 \\
\hline 7 & NT & 0 & ACR & 60 & IOBE & 450 & 72.5 \\
\hline 8 & NT & 0 & ACR & 60 & IOIE & 535 & 89.9 \\
\hline 9 & NT & 0 & ACR & 60 & UIE & 900 & 99.9 \\
\hline 10 & REFCL & 4000 & ACR & 60 & IOBE & 450 & 86.0 \\
\hline 11 & REFCL & 4000 & ACR & 60 & IOIE & 535 & 95.5 \\
\hline 12 & REFCL & 4000 & ACR & 60 & UIE & 900 & 99.9 \\
\hline
\end{tabular}

\section{Results}

The mathematical model for the case study was coded in Python and was solved using the CPLEX solver. The results of MC in terms of proposed zone substations treatments (Z.S.T.), proposed sections treatments (S.T.), and proposed poles treatments (P.T.) for reducing the risks of bushfires from $10 \%$ to $90 \%$ are summarized in Figure $3 \mathrm{a}$ ) and b). Recall that consequence is currently being treated as constant across the demonstration network. These results therefore correspond to ignition reductions, where the full Victorian power network can record a few hundred to over a thousand ignitions in a single year. For reductions above 90\%, REFCL installations were needed. Between $10 \%$ and $60 \%$ only REFCL and ACR protection was needed. Beyond $60 \%$ individual pole treatments became necessary. Beyond $60 \%$ there was diminishing return on investments.

In order to investigate the effects of poles, sections, and zone substation treatments on risk reduction, a second model, Minimum Risk (MR) was considered. This model's objective function minimized bushfire risk across the network subject to cost constraints. The total spend allowed in this model was $\$ 18.48$ million, the amount

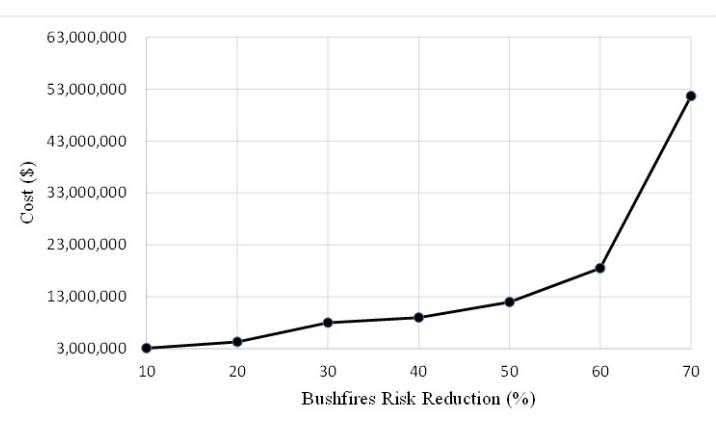

(a). $10 \%$ to $60 \%$ risk reduction

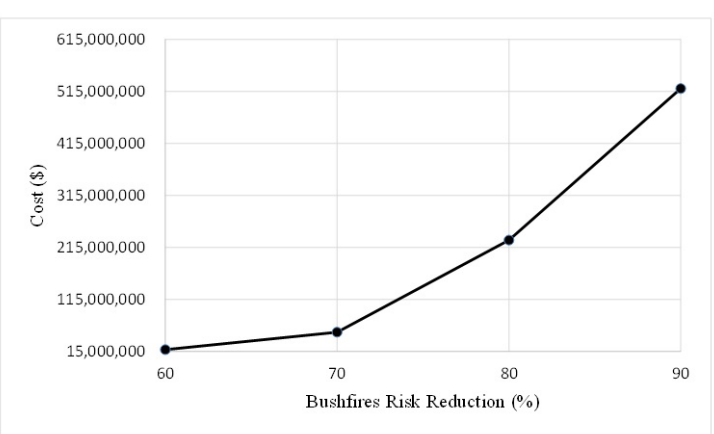

(b). $60 \%$ to $90 \%$ risk reduction

Figure 3. Network improvement costs for bushfire risk reductions between $10 \%$ and $90 \%$. Note that the demonstration network is only $5 \%$ to $10 \%$ of the size of the true Victorian network in terms of the number of distribution assets represented. 


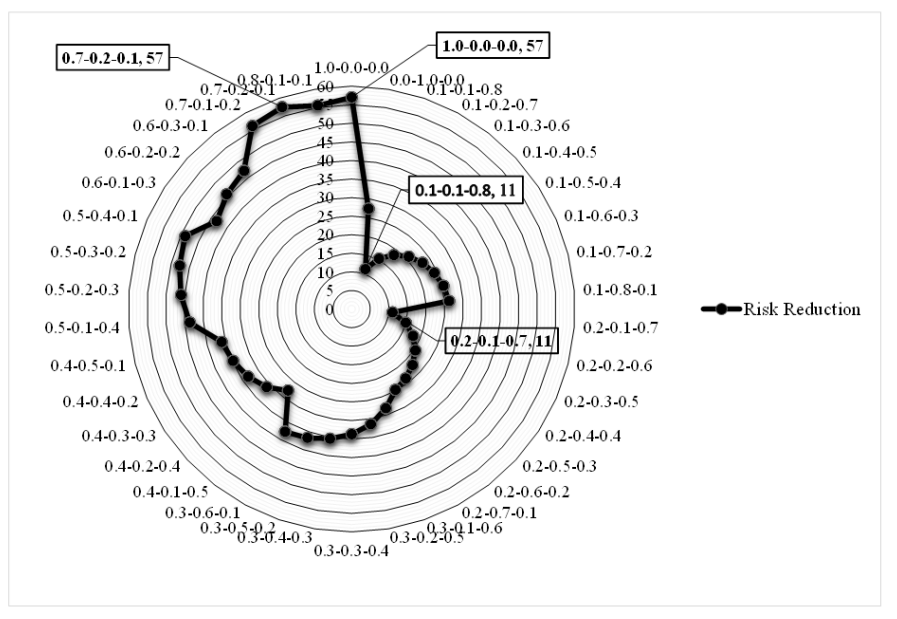

Figure 4. Bushfire risk reductions given by the MR model with cost constraints. Numbers are indicated in a substation, section, poles order as proportion of money spent. Substation spending is increased in a clockwise direction. Best and worst reduction spending combinations are highlighted in boxes.

required for a $60 \%$ reduction in the MC results. Figure 4 graphs results on a circular axis indicating the proportion of substations, sections, and poles financed during different network improvements. These results indicate that focusing money spent on substations and sections yields better reductions than paying for line and pole treatments.

\section{Conclusions}

An integer program model was used to find optimum asset combinations for improvement of a powerline network symbolic of those used in Victoria. Results indicate that treatments applied to zone substations and treatments are required to reduce risks to a $60 \%$ reduction target. Costs rise dramatically to achieve higher risk reductions. We also identified that combinations of zone substation and section treatments achieve better risk reduction results compared to section and pole treatments. An alternative fiscally reasonable approach to consider in future work would be to target pole treatments to poles with a high likelihood of faults or in high consequence areas. Decreasing the risk for the whole network is unrealistically costly.

\section{REFERENCES}

Altay, N. and W. Green (2006). OR/MS research in disaster operations management. European Journal of Operational Research 175(1), 475-493.

Dunstall, S., C. Huston, G. Towns, A. Stephenson, and C. Miller (2015). PBSP risk reduction model. Technical report, CSIRO Digital Productivity.

Energy Safe Victoria (2011). Powerline bushfire safety taskforce final report. Technical report, Energy Safe Victoria (ESV).

Higgins, A., S. Whitten, A. Slijepcevic, L. Fogarty, and L. Laredo (2011). An optimisation modelling approach to seasonal resource allocation for planned burning. International Journal of Wildland Fire 20(2), 175-183.

Hof, J., P. N. Omi, M. Bevers, and R. D. Laven (2000). A timing-oriented approach to spatial allocation of fire management effort. Forest Science 46(3), 442-451.

Minas, J., J. Hearne, and D. Martell (2013). An integrated optimization model for fuel management and fire suppression preparedness planning. Annals of Operations Research, 1-15.

Minciardi, R., R. Sacile, and E. Trasforini (2009). Resource allocation in integrated preoperational and operational management of natural hazards. Risk Analysis 29(1), 62-75.

Teague, B., R. McLeod, and E. Trasforini (2010). 2009 victorian bushfires royal commission - final report. Technical report, Parliament of Victoria. 